\title{
Midline Spikes in Children, is Long-Term Treatment Needed?
}

\author{
Juan Pablo Appendino ${ }^{1^{*}}$ and Bashir Brebesh ${ }^{2}$ \\ ${ }^{1}$ Department of Paediatrics, University of Calgary, Canada \\ ${ }^{2}$ Department of Internal Medicine, Health Sciences Centre, Canada
}

Submission: March 02, 2017; Published: July 19, 2017

*Corresponding author: Juan Pablo Appendino, MD, Clinical Associate Professor, Department of Paediatrics, Cumming School of Medicine, University of Calgary Pediatric Epilepsy and Child Neurology, Alberta Children's Hospital, 2888 Shaganappi Trail NW, Calgary, AB T3B 6A8, Canada, Pager: 403-212-8223 \#14691, Tel: 403-955-7816; Fax: 403-955-7609; Email: jp.appendino@albertahealthservices.ca

\begin{abstract}
Introduction: Isolated midline spike (IMS) is an unusual finding in the paediatric electroencephalogram (EEG). Its significance in developing epilepsy, therefore needing long term anti-seizure medications, is unknown. We hypothesized that in previously healthy children there could be no need of long term commitment to anti-seizure medications despite the presence of IMS on their EEG.

Methods: This is a descriptive, single centre, retrospective cohort study of consecutive pediatric patientspresented to our neurophysiology laboratory from April 2009 to March 2011 in whose first EEG showed IMS. Neurophysiology database was queried for key words "midline spikes". Inclusion and exclusion criteria were met. Local research ethical board approval was obtained.

Results: 241 EEGs were retrieved from the query to be reviewed. 60 patients met inclusion criteria. 45 patients had seizures and 15 had other reasons to request an EEG (i.e. ataxia, ADHD, behaviour disorders). 33 patients had normal neurological examination and neuroimaging and 27 did not. Twenty six out of the 33 normal patients had follow up. Only 2 developed epilepsy. Twenty two of the 27 abnormal patients had follow-up. Seventeen developed epilepsy (OR 41; 95\% CI 7- 236; p<0.0001).
\end{abstract}

Conclusion: Despite the small sample group, these results suggest that the vast majority of patients with IMS who are normal, are unlikely to develop epilepsy. These findings support a conservative approach when considering anti-seizure medication.

Keywords: Isolated midline spikes; Children; Seizure; Epilepsy; Developmental delay; Neurological examination; Neuroimaging; EEG

\section{Background}

Isolated midline spike (IMS) is an unusual finding on the electroencephalogram (EEG). Young et al. [1] reported in 2008 that only $17(0.21 \%)$ out of 7,929 EEGs performed in adults and children had IMS [1]. Vendrame et al. [2] also reported similar figures in the pediatric population. $65(0.54 \%)$ out of 12,000 EEGs were found to be consistent with IMS [2]. IMS are known to mainly occur in children. Pedley et al. [3] reported 78\% while Nelson et al. [4] reported $71 \%$ of patients with IMS were younger than 18 year-old .IMS has been highly correlated with seizures and epilepsy. It has been published that $73 \%$ to $81 \%$ of patients with IMS had a single or multiple seizures [4,5]. Bagdorf et al. [6] found that 14 (48\%) out of 29 patients with IMS and epilepsy had poor seizure control [6] and Vendrame et al. [2] reported that $61 \%$ of their patients with IMS had epilepsy [2]. IMS and vertex waves (a normal sleep feature) are at times difficult to differentiate; however, vertex waves are not seen during awake state.
Previous studies included both a minority of patients with normal and a majority of patients with abnormal EEG background activities, abnormal neurologic examination, and abnormal neuroimaging. This explains that conclusions were mainly focused on those who presented the previously mentioned abnormalities $[2,6]$. They also included IMS recorded during awake and sleep states with the exception of Vendrame et al. [2] who only included IMS during awake state in order to clearly differentiate them from vertex waves [2]. To our knowledge, there is no study that focused on normal patients with IMS on their EEG and their risk of developing epilepsy as well as their need of long term commitment to anti-seizure medications. We postulate that the finding of IMS represent a benign finding and does not carry an increased risk of developing epilepsy on children's EEG who present with a seizure but neurologically are normal along with normal neuroimaging. Therefore treatment with daily anti-seizure medicationsis not required. 


\section{Open Access Journal of Neurology \& Neurosurgery}

\section{Methods}

This is a single-centre retrospective cohort study. The period studied was from April 2009 to March 2011. The database of the neurophysiology laboratory at the Children's Hospital of Winnipeg was queried for midline spikes. All EEGs during the assessed timespan were recorded according to International 1020 System of electrode placement, which includes 19 electrodes and $\mathrm{Cz}^{\prime}$ as reference $\left(\mathrm{Cz}^{\prime}\right.$ is located half distance between $\mathrm{Cz}-\mathrm{Fz}$ electrodes). A recording of at least 20 minutes including at awake state was considered appropriate. We identified EEGs which were reported to have only IMS without any other background or interictal abnormalities during awake state. If present during sleep, IMS had to be clearly differentiable from normal sleep patterns. IMS had to be present in both referential and bipolar montages.IMS was defined as a negative spike or spike and slow wave discharge with maximum negativity over $\mathrm{Fz}, \mathrm{Cz}$ or $\mathrm{Pz}$. (Figure 1). The identified EEGs were then reviewed by a certified neurophysiologist (JPA) in a blindly manner to gender, outcome, physical examination and neuroimaging results. Patients whose EEG traces were confirmed to have IMS were recruited. Their hospital charts were then reviewed and data was anonymously collected in a systematic fashion over a pre-determined datacapture sheet. Descriptive results were obtained upon the latest follow up appointment. Patients without follow up were not included in outcome analysis.

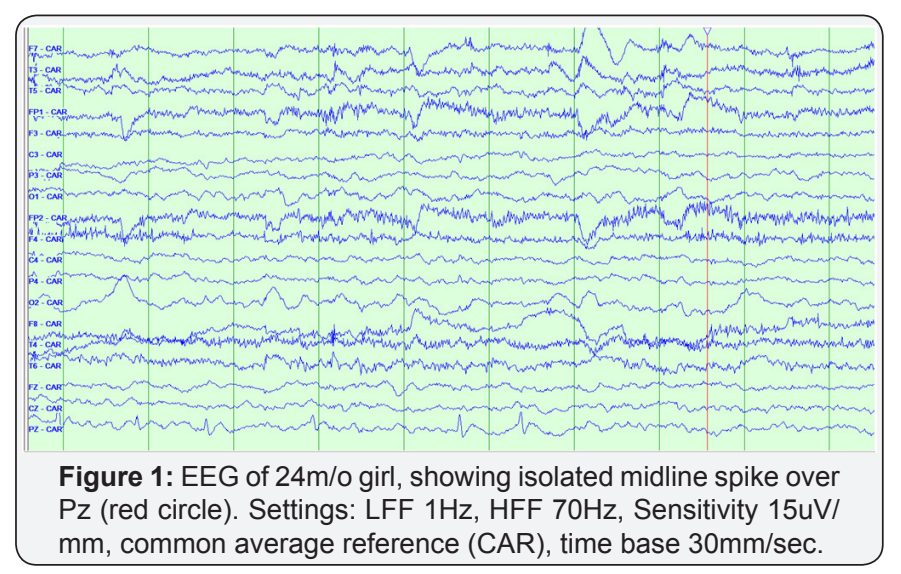

Patients were divided into two groups according to their physical examination, development status and neuroimaging results. Those with only IMS on their EEG but otherwise normal findings were included into group "N"; while those with other abnormalities apart from the IMS on their EEGs were grouped into group "A". Patients whose follow up information was available were further divided into those who develop epilepsy (group "N-E" and group "A-E", as for normal-epilepsy and abnormalepilepsy respectively) or into those who did not develop epilepsy (group "N-N" and group "A-N", as for normal-no epilepsy and abnormal-no epilepsy). Epilepsy was defined as the occurrence of two or more unprovoked seizures separated in more than 24 hours apart as per ILAE definition $[7,8]$. Statistical analysis for nonparametric testing were performed. This included the odds ratio calculations for the assessment of the two groups with and without epilepsy, $\chi 2$ test, and Mann-Whitney U test. Statistical analysis was performed with SPSS software Version 20 (in the university of Manitoba library).

\section{Results}

\section{EEG analysis}

There was a total of 1576 EEG recorded during the two years study period. 266 out of those were associated with IMS in the database. After detailed review, 60 EEG met the inclusion criteria (Figure 2).

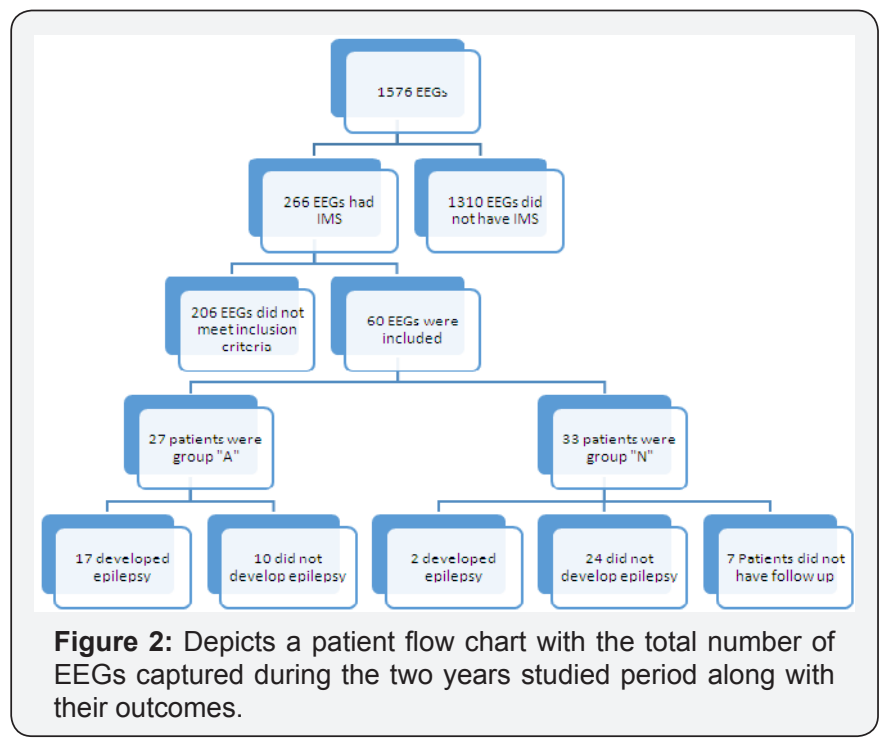

\section{Clinical Data and seizure patterns}

These 60 patients ranged in age from 11 months to 15 years old; with mean age of 6.4 years. There were 23 females (38.3\%) and 37 males (61.7\%). Clinical seizure was the most frequent reason for referral to the EEG labs (45 patients), other reasons for referral (14 patients) included burst of aggression, episodic ataxia, inattention, an episode of dizziness \& sweating, infantile gratification syndrome, confusion and migraine with aura. Probable etiologies for the seizures were found in 18, including neurocutaneous syndromes, brain tumors, SCNA1 mutation, fetal alcohol syndrome, agenesis of corpus callosum, bacterial meningitis, subdural hematoma, major head injury, cerebral palsy, juvenile myoclonic epilepsy, perinatal stroke, and hemolytic uremic syndrome. Three patients had a family history of seizures. Nineteen patients had seizures with upper respiratory tract infections or gastroenteritis.

Abnormal (focal) neurological examinations were noted in 27, and abnormal brain imaging (brain MRI and or brain CT) was found in 17 . The abnormalities included mesial temporal sclerosis, cortical tubers, tumor, gliosis and encephalomalacia post traumatic brain injury, hemorrhagic contusion, what matter rarefication and agenesis of corpus callosum, subdural 


\section{Open Access Journal of Neurology \& Neurosurgery}

hematoma, cortical dysplasia and cerebellar atrophy, asymmetry in the size of cerebellar hemispheres, and gliosis due perinatal ischemic lesion. Seizures were described as generalized tonic clonic in 30 patients, 7 patients had focal motor seizures, 3 patients had absence seizures, 3 patients had atonic seizures and 2 patients had focal twitching with secondary generalization.

Forty eight patients had follow up in the pediatric neurology outpatient clinic, period of Follow up ranged between 6 months to 5 years; with mean period of 1.5 years. Twenty seven patients were neurologically abnormal on examination and/ or by neuroimaging. Fourteen patients had presentation other than seizures. Thirty three patients had a normal neurological and normal brain imaging. Twenty six had follow up and only 2 patients developed subsequent seizures. Interestingly, the follow up EEG of these 2 patients showed epileptiform discharges in locations other than the midline region. One had generalised discharges, and was diagnosed with juvenile myoclonic epilepsy, with the presence of both generalised tonic clonic seizures and myoclonic jerks. The other one had focal frontal discharges. In remainder of 24 patients, three patients were treated with anti-seizure medication, nineteen had follow up EEG, 9 showed normal EEG, and 10 showed persistence of midline spikes.

Out of the 27 patients with abnormal neurological examinations and/or abnormal imaging 17 had subsequent seizures. The calculated odds ratio of developing subsequent seizures after finding of isolated Midline spikes, in patients with abnormal neurological examinations and/ or abnormal brain imaging was (OR 41; 95\% CI 7- 236; p<0.0001). Fourteen patients had initial presentation other than seizures .All had normal neurological exam and normal brain imaging, they did not subsequently develop epilepsy.

\section{Discussion}

Table 1: Patients abnormal (focal) neurological exam and or abnormal Neuroimaging.

\begin{tabular}{|c|c|c|c|c|c|c|c|}
\hline No & Age & Sex & presentation & Brain imaging & Epilepsy & Notes & $\mathbf{F} / \mathbf{u}$ \\
\hline 1 & $5 \mathrm{Ys}$ & $\mathrm{F}$ & GTC Sz & Lt Mesial temporal sclerosis & yes & & 5 years \\
\hline 2 & $18 \mathrm{Ms}$ & M & GTC Sz & Normal & No & DD & 4 years \\
\hline 3 & 2 Ys & $\mathrm{F}$ & Absence Sz & Cortical tuber & yes & & 4 years \\
\hline 5 & $2 \mathrm{Ys}$ & $\mathrm{F}$ & Focal Sz & Brain tumor & Yes & & 2 years \\
\hline 6 & $2.5 Y s$ & $\mathrm{~F}$ & GTC Sz & Normal & Yes & DD & 3 Years \\
\hline 7 & $1 \mathrm{Ys}$ & M & Atonic & Rt frontal gliosis & Yes & & 3 years \\
\hline 8 & 2 Ys & M & GTC Sz & Normal & Yes & SCNA1 Mutation & 2 years \\
\hline 9 & $1 \mathrm{Y}$ & M & Focal Sz & Old hemorrhage & Yes & & 4 years \\
\hline 10 & 3 Ys & M & GTC Sz & Corpus callosum Agenesis & Yes & & 5 years \\
\hline 11 & $4 \mathrm{Ys}$ & M & GTC $S z$ & Brian atrophy & Yes & FASD & 2 years \\
\hline 12 & $13 \mathrm{Ms}$ & M & GTC Sz & Subdural hematoma & No & & 2 years \\
\hline 13 & $1 \mathrm{Ys}$ & M & Atonic & Focal gliosis & Yes & & 2 years \\
\hline 14 & $6.5 \mathrm{Ys}$ & M & GTC sz & Normal & No & DD & 3 years \\
\hline 15 & 14 Ys & $\mathrm{F}$ & Focal Sz & Cortical dysplasia & Yes & & 3 years \\
\hline 16 & $16 \mathrm{Ms}$ & $\mathrm{F}$ & GTC Sz & Megalohencephaly & Yes & & 5 years \\
\hline 17 & 6 Ys & M & $\mathrm{BOA}$ & Normal & No & $\mathrm{DD}$ & 1 year \\
\hline 18 & 12 Ys & M & Focal Sz & Craniotomy & Yes & Post Trauma & 3 years \\
\hline 19 & $8 \mathrm{Ys}$ & M & Focal Sz & Cortical tubers & Yes & NF1 & 5 years \\
\hline 20 & $11 \mathrm{Ms}$ & M & GTC Sz & Perinatal stroke & Yes & $\mathrm{CP}$ & 2 years \\
\hline 21 & 13 Ys & M & GTC $S z$ & Frontal old contusion & No & & 3 years \\
\hline 22 & $5 \mathrm{Ys}$ & M & Atonic & Whit matter rarefication & yes & HIE & 3 years \\
\hline 23 & $1 \mathrm{Y}$ & M & GTC Sz & Perinatal stroke & yes & $\mathrm{CP}$ & 5 years \\
\hline
\end{tabular}

Abbreviations: HIE : Hypoxic Ischemic Encephalopathy; FASD: Fetal Alcohol Syndrome; CP: Cerebral Palsy; NF: Neuro Ffibromatosis; BOA: Burst of Aggression, DD: Developmental Delay; IGS: Infantile Gratification Syndrome

Previous studies focused primarily on clinical features and electroencephalographic characteristics of patients with midline spikes [1-3]. Vendrame et al. [2] studied children and young adults with IMS [9]. She compared, children who developed epilepsy with those who did not. However, they omitted the difference between previously normal and abnormal patients. A Major shortcoming of previous retrospective studies is that they included patients with abnormal EEG background, generalized spike-wave or other foci, as long as the midline spikes were the most prominent abnormality, they concluded that there is an increased association of midline spikes and developing epilepsy. Nine of the fourteen patients the Pedley et al. [3] reported had abnormal EEG background (4 diffusely slow, 3 had lateralized slowness, and in one the slowness was confined 


\section{Open Access Journal of Neurology \& Neurosurgery}

to the posteriorly) as well 3 patients had generalized burst of bilaterally synchronous spike and wave paroxysms in addition to the midline spikes [1]. We included only patients with isolated midline spikes. On the other hand; even in the studies with only midline spikes, the study group had patients with abnormal neurological exam and abnormal neuroimaging in the study group. Bagdorf \& Lee [6] reported in 199357 with IMS, 29 had follow up, 14 developed epilepsy. However, eight had abnormal neurological examination, and six abnormal neuroimaging [10] (Table 1). No study to our knowledge discussed the prognosis of isolated midline spikes in regard of development of epilepsy in children with normal neurological examination and normal brain imaging, and no study to our knowledge discussed the management with anti-seizure medications in this particular group of patients (Table 2).

Table 2: Patients with normal neurological examination and normal brain imaging.

\begin{tabular}{|c|c|c|c|c|c|c|c|c|c|}
\hline No & Age & Sex & Presentation & Fever & $\mathbf{F} / \mathbf{u}$ & F/U EEG & $\begin{array}{l}\text { Follow up } \\
\text { Duration }\end{array}$ & Epilepsy & AED \\
\hline 1 & 13 Ys & $\mathrm{F}$ & Absence Sz & No & yes & Unavailable & 2 years & No & No \\
\hline 2 & 4 Ys & M & GTC Sz & No & yes & Midline spikes & 3 years & No & No \\
\hline 3 & $8 \mathrm{Ys}$ & M & Burst of aggression & No & yes & Unavailable & 6 months & No & No \\
\hline 4 & $11 \mathrm{Ms}$ & $\mathrm{F}$ & GTC Sz & yes & yes & Midline spikes & 2 years & No & No \\
\hline 5 & $5 \mathrm{Ys}$ & M & Episodic ataxia & No & yes & Normal & 2 Years & No & No \\
\hline 6 & $1 \mathrm{Y}$ & $\mathrm{F}$ & GTC Sz & yes & yes & Midline spikes & 2 Years & No & No \\
\hline 7 & $18 \mathrm{Ms}$ & M & GTC Sz & No & yes & Midline Spikes & 4 Years & No & Yes \\
\hline 8 & $5 \mathrm{Ys}$ & $\mathrm{F}$ & GTC Sz & Yes & yes & Normal & 3 Years & No & No \\
\hline 9 & 13 Ys & $\mathrm{F}$ & Absence Sz & No & yes & Midline spikes & 2 years & No & No \\
\hline 10 & $2.5 \mathrm{Ys}$ & $\mathrm{F}$ & GTC Sz & Yes & Yes & Midline spikes & 6 months & No & No \\
\hline 11 & $3 \mathrm{Ys}$ & M & Inattention & No & Yes & Unavailable & 3 months & No & No \\
\hline 12 & $6.5 \mathrm{Ys}$ & M & Dizziness & No & Yes & Normal & $1 \mathrm{Y}$ & No & No \\
\hline 13 & $8 \mathrm{Ms}$ & $\mathrm{F}$ & GTC Sz & Yes & Yes & Normal & $2 \mathrm{Ys}$ & No & Yes \\
\hline 14 & $1 \mathrm{Y}$ & M & GTC Sz & No & yes & Normal & 2 years & No & No \\
\hline 15 & 15 & $\mathrm{~F}$ & Absence & No & yes & Generalized Epileptiform discharges & 3 Year & Yes & Yes \\
\hline 16 & $6.5 \mathrm{Ys}$ & M & GTC Sz & No & yes & Midline spikes & 3 Years & No & Yes \\
\hline 17 & 3 Ys & M & GTC Sz & Yes & Yes & Normal & 3 Years & No & Yes \\
\hline 18 & 2 Ys & $\mathrm{F}$ & GTC Sz & No & Yes & Midline Spikes & 2 Years & No & No \\
\hline 19 & 3 Ys & $\mathrm{F}$ & GTC Sz & Yes & Yes & Midline spikes & 2 years & No & No \\
\hline 20 & 11 Ys & M & Focal Sz & No & Yes & Other Epileptiform discharges & 5 years & yes & yes \\
\hline 21 & $3 \mathrm{Ys}$ & $\mathrm{F}$ & GTC Sz & No & yes & Unavailable & 2 years & No & No \\
\hline 22 & $1 \mathrm{Y}$ & M & GTC Sz & Yes & Yes & Midline Spikes & 2 years & No & No \\
\hline 23 & 2 Ys & $\mathrm{F}$ & GTC Sz & No & Yes & Normal & 2 years & No & No \\
\hline 24 & $1 \mathrm{Y}$ & $\mathrm{F}$ & Focal Sz & No & yes & Normal & 3 years & No & No \\
\hline 25 & $7 \mathrm{Ys}$ & M & Migraine & No & Yes & Normal & 6 months & No & No \\
\hline 26 & $6 Y s$ & M & Burst of aggression & No & yes & Unavailable & 1 Year & No & No \\
\hline
\end{tabular}

Among our 60 cases with IMS, 33 were neurologically normal twenty two had follow up from those only 2 developed epilepsy, these 2 patients had follow up EEG that showed epileptiform discharges in other locations. In the neurologically abnormal (group 2) 27 patients, 17 had subsequent epilepsy (OR 41; 95\% CI 7- 236; $\mathrm{p}<0.0001$ ). Period of Follow up ranged between 6 months to 5 years; with mean period of 1.5 years [11].

The mechanism of the midline spikes is uncertain. Patients with midline/ parasagittal legions could develop midline spikes.
McLachlan and Girvin [12] described the electroencephalographic features Interictal midline spikes from penicillin foci located induced in mesial cortex of cats and compare it with 16 patients with interictal midline spikes. They demonstrated that midline spikes originate focally from the mesial cortex [8]. We identified 14 patients with IMS, who have non seizure presentation (Table 3 ), indicating the midline spikes are not specific for seizures. We have small number, and we did not have long follow up period for all of our patients, a larger sample, and long term clinical and EEG follow up can provide more information [13]. 


\section{Open Access Journal of Neurology \& Neurosurgery}

Table 3: Patients with presentation other than clinical seizures.

\begin{tabular}{|c|c|c|c|c|}
\hline No & sex & Age & Presentation/ Diagnosis & Epilepsy \\
\hline 1 & M & 8 Ys & Burst of Aggression/ ADHD, & No \\
\hline 2 & M & 5 Ys & $\begin{array}{c}\text { Episode of unsteadiness/ Episodic } \\
\text { ataxia }\end{array}$ & No \\
\hline 3 & M & 3 Ys & Autism spectrum disorder & No \\
\hline 4 & M & 9 Ys & $\begin{array}{c}\text { Burst of aggression/ Asperger } \\
\text { syndrome }\end{array}$ & No \\
\hline 5 & M & 2.5 Ys & Prolonged staring/ Developmental & delay \\
\hline 6 & M & 6.5 Ys & Episode of sweating \& Dizziness & No \\
\hline 7 & M & 4 Ys & Infantile gratification syndrome & No \\
\hline 8 & M & 5 Ys & ADHD & No \\
\hline 9 & F & 2 Ys & Confusion & No \\
\hline 10 & F & 6 Ys & Inattention / ADHD & No \\
\hline 11 & M & 9 Ys & Burst of aggression & No \\
\hline 12 & M & 7 Ys & Migraine with Aura & No \\
\hline 13 & M & 4 Ys & Staring / Autism spectrum & No \\
\hline 14 & M & 6 Ys & Burst of aggression & No \\
\hline
\end{tabular}

\section{Conclusion}

Our data suggests that in patients with a single initial seizure in whom the EEG is abnormal showing IMS; however, a normal neurologic examination and a normal neuroimaging are found, then the most prudent course of action would be a conservative approach without initiation of anti-seizure medications. Midline spikes could also be present in patients with non-seizure presentation. This is an already reported finding but it reinforces the awareness.

\section{Acknowledgment}

The authors would like to thank Christina Anderson in the Children's Hospital of Winnipeg Neurophysiology laboratory for her valuable help during EEG query and analysis

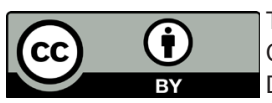

This work is licensed under Creative Commons Attribution 4.0 Licens DOI: 10.19080/OAJNN.2017.04.555648

\section{Ethical Approval}

This study was approved by Research ethics board University of Manitoba.

\section{References}

1. Yong T, Chayasirisobhon S, Yoshina JS, Tin SN, Tehrani K, et al. (2008) Midline spikes. Clin EEG Neurosci 39(1): 28-32.

2. Vendrame M, Tracy M, Das R, Duffy F, Loddenkemper T, Kothare SV (2010) Clinical correlations of midline spikes in children. Epilepsy Behav 18(4): 460-465.

3. Pedley TA, Tharp BR, Herman K (1981) Clinical and electroencephalographic characteristics of midline parasagittal foci. Ann Neurol 9(2): 142-149.

4. Nelson KR, Brenner RP, de la Paz D (1983) Midline spikes. EEG and clinical features. Arch Neurol 40(8): 473-476.

5. Pourmand RA, Markand ON, Thomas C (1984) Midline spike discharges: clinical and EEG correlates. ClinElectroencephalogr 15(4): 232-236.

6. Bagdorf R, Lee SI (1993) Midline spikes: is it another benign EEG pattern of childhood? Epilepsia 34(2): 271-274.

7. Fisher RS, Boas WV, Blume W, Elger C, Genton P, et al. (2005) Epileptic seizures and epilepsy: definitions proposed by the International League against Epilepsy (ILAE) and the International Bureau for Epilepsy (IBE). Epilepsia 46(4): 470-472.

8. Ehle A, Co S, Jones MG (1981) Clinical correlates of midline spikes. An analysis of 21 patients. Arch Neurol 38(6): 355-357.

9. Molaie M (1985) Mid-line epileptiform activity in adult electroencephalograms. Clin Electroencephalogr 16(1): 54-61.

10. Konishi T, Naganuma Y, Hongou K, Murakami M, Yamatani M, et al. Clinical and EEG evaluation of mid-line spikes in childhood. No To Shinkei 40(12): 1189-1193.

11. McLachlan RS, Girvin JP (1989) Electroencephalographic features of midline spikes in the cat penicillin focus and in human epilepsy. Electro encephalogr Clin Neurophysiol 72(2): 140-146

12. Amit R, Crumrine PK (1993) Ictal midline epileptiform discharges. Clin Electroencephalogr 24(2): 67-69.

13. Kutluay E, Passaro EA, Gomez-Hassan D, Beydoun A (2001) Seizure semiology and neuroimaging findings in patients with midline spikes. Epilepsia 42(12): 1563-1568.

\section{Your next submission with Juniper Publishers will reach you the below assets}

- Quality Editorial service

- Swift Peer Review

- Reprints availability

- E-prints Service

- Manuscript Podcast for convenient understanding

- Global attainment for your research

- Manuscript accessibility in different formats

( Pdf, E-pub, Full Text, Audio)

- Unceasing customer service

Track the below URL for one-step submission https://juniperpublishers.com/online-submission.php 